\title{
Lung cancer patient advocacy and participatory medicine
}

\author{
Kim Norris
}

\begin{abstract}
Kim Norris answers questions on the role of the patient advocate within a participatory medicine system.
\end{abstract}

\section{Introduction}

Kim Norris (Figure 1) is the President and co-founder of the Lung Cancer Foundation of America (LCFA) [1], an advocacy group for lung cancer patients that seeks to raise research funding and the national profile of lung cancer to increase support for research. She became a patient advocate after her husband died of lung cancer at the age of 47 in 1999, following a suggestion from her husband's clinician. In 2007, after experiencing difficulties in obtaining public funds for research on lung cancer, she and two lung cancer survivors, Lori Monroe and David Sturges, co-founded LCFA to raise funds from the private sector. Kim Norris shares her views on how genomic profiling is changing the role of patients in healthcare.

\section{What are the challenges and highlights in your role as} President of the Foundation?

The highlights are always the patients and their families. When I hear a success story or I hear that we have made a difference, whether it is in a patient's life or their family's life, that just touches me to the core and makes me realize that this is what I should be doing.

The challenges are enormous. Lung cancer is underfunded, both from a public and a private standpoint. It is very difficult to obtain research funding. This is probably for two reasons. The first is the stigma associated with lung cancer: there is a belief that the patients did it to themselves, and many people think, 'Well, if you have

Correspondence: KNorris@LCFAmerica.org

Lung Cancer Foundation of America, 15 S. Franklin St, New UIm, MN 56073, USA lung cancer, then you smoked.' This is despite over $60 \%$ of new lung cancer diagnoses being in non-smokers. Overcoming the societal bias about lung cancer is very difficult.

The second reason I think it is hard to get funding for lung cancer research is that the 5-year survival rate is only $15 \%$, compared with, for example, $88 \%$ and $99 \%$ for breast or prostate cancer, respectively. Both breast and prostate cancer have a large number of survivors who raise awareness of these diseases; lung cancer does not have a legion of survivors. So it's really up to the family members and friends to make a difference.

What is a patient advocate and how do you become one? There are many different types of patient advocate. A patient advocate can range from someone who works with patients and helps them through chemotherapy to someone who works with local hospitals to make sure patients are getting the care, the treatment and the support that they need. Being a patient advocate can also mean working with researchers and helping set up clinical trials, making sure that the recruiting process is appropriate and that the patient's point of view is considered. Patient advocacy can also be activism for research funding or getting involved with organizations to raise awareness, or to help educate the public. So patient advocacy comes in all shapes, sizes and flavors.

For example, my co-founder, Lori Monroe, was a registered nurse. She became a patient advocate after being diagnosed with stage IV lung cancer in 2001: she used her own medical knowledge and experience to advocate for herself in a way that few lung cancer patients could. She recognized the importance of medical research in the development of new options for lung cancer patients, and became a patient advocate with the National Cancer Institute's (NCI) Lung Specialized Programs of Research Excellence (SPORE) initiative at Vanderbilt University and with other programs. 


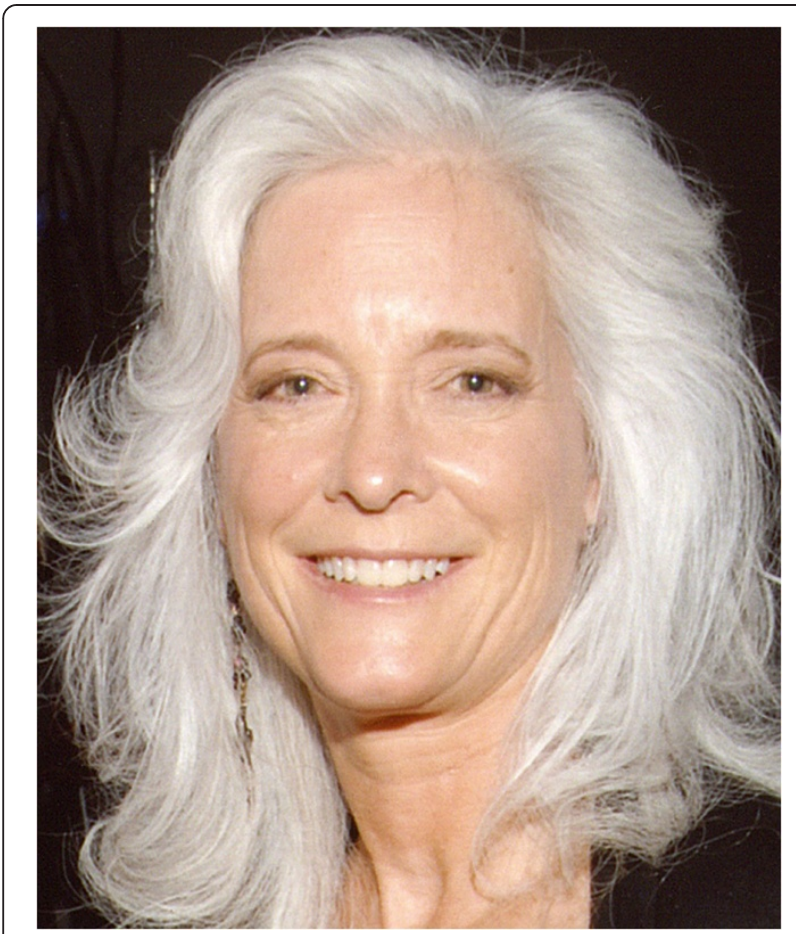

Figure 1 Kim Norris.

How will the role of cancer patient advocacy groups change as we move towards genomic profiling and personalized medicine?

The role of the patient advocacy groups is more critical than ever before. For example, in lung cancer, targeted therapy and personalized medicine are progressing rapidly, and this is very exciting. However, genomic information is mostly confined to academics - $85 \%$ of patients are treated in community hospitals and do not have access to academic centers. Getting one's tumor tested for its genomic profile is essential for assessing treatment options, but it is not a widespread practice in non-academic centers. I think that this is where advocacy organizations can play a role to help educate patients, to liaise with the research community and patients, and make people aware of their options. They can also work with community hospitals, organizations and doctors to help inform them about the importance of getting their tumor tested. I think the role of the advocacy organizations is pivotal.

Will advocacy groups for non-cancer diseases also experience this change?

I think the role of patient advocacy groups is important whether it is lung cancer or any other disease. All advocacy organizations can play a very important role in educating themselves and their patients, working closely with researchers, and also stepping up in case there are any regulatory issues. I think this applies to just about any disease-specific advocacy organization.

\section{How has lung cancer research been transformed by genomics profiling?}

In the past 5 years I've started to get really excited about genomic profiling. The Cancer Genome Atlas project has provided a wealth of genomic information, and luckily lung cancer was one of the first cancers that was investigated by researchers [2]. Before, if you had lung cancer, you would undergo chemotherapy and it was a one-size-fits-all approach, whereas now we realize that tumors are different from person to person [2-5]. By identifying those unique characteristics, it is possible to develop and create targeted therapies, thereby increasing the chance of killing the tumor. That is really gamechanging in the world of lung cancer, and I think in the world of cancer in general.

The other important change is in clinical management. As a result of genomic profiling, we are shifting the way we look at cancer in general. Cancers are now thought of as chronic diseases, and rather than necessarily curing them, we are looking at ways of taking medication to manage the disease for the rest of a patient's life.

\section{Do you think the relationship between patient advocacy groups, researchers and clinicians is changing?}

Yes, because each group has to be aware of new research and changes that are happening so quickly. There is also a difference in mind set, which is bypassing the organspecific view of cancers to an idea that they are molecular diseases. This has an impact on models for raising research funds.

\section{As a patient advocate, what are your thoughts on participatory medicine?}

The model of participatory medicine is coming into its own. It is now critical for patients to be more involved in their healthcare and more engaged in their disease. The days when your primary care physician or the doctor who you have been seeing for 30 to 40 years knows it all are gone. The patient needs to go to their doctor armed with the right questions and information. I also think that the traditional paradigm of a single doctor managing a disease is changing; it's likely to be a team of doctors who are working with a single patient.

As an example, recently I was on a panel with pathologists, and the message I was trying to give is that pathologists were traditionally the ones in the background that analyzed the tissue and reported back. The patients have no idea who or what a pathologist is. But now a pathologist plays a really critical role in the whole notion of genomic profiling. They are part of the patient's team, and they should be more involved with the patients. In fact, 
they may be more educated about unique mutations than the general clinicians who are treating the patients.

\section{What do you think are the remaining challenges for the} implementation of this model of healthcare?

I think this model of healthcare still has many challenges to overcome. I know that this not true for all countries, but in the United States a lot of it has to do with the issue of insurance and healthcare coverage. So, I think that the change is happening faster than the societal model that goes with it.

In the case of genomic profiling for lung cancer, more than 200 genomic mutations have been identified. So, do clinicians perform a genomic profile searching for all of them, or only for the top few, even though there are not necessarily FDA-approved treatments but many exciting clinical trials going on to target them? Part of the issue is what do you test for and who is going to pay for it.

Regarding participatory medicine, the more a patient can be informed and knowledgeable about their disease the better; the more a patient can question and challenge their doctor, get second, third or fourth opinions, and have a key role in their own healthcare the better. I think these are the important aspects for the future of participatory medicine.

\section{Abbreviation \\ LCFA: Lung cancer foundation of America.}

\section{Competing interests}

The author declares that she has no competing interests.

\section{Acknowledgements}

Shortly after this article was written, Lori Monroe, one of LCFA's co-founders, died, 12 years after she was first diagnosed with lung cancer. Lori's loss is profound, not just for me, personally, but for the entire lung cancer advocacy community. She was a tireless and selfless advocate for lung cancer patients. LCFA and the lung cancer community have lost a dear friend, mentor, warrior and someone who was extraordinary in all of our eyes. We will remember Lori always for her energy, her passion and her commitment, but also for her incredible warmth, charm, humor and compassion. Her memory will continue to be a source of guidance and inspiration for us, and we will continue to work as diligently as she did to keep patients alive and healthy.

Published: 30 January 2014

\section{References}

1. Lung Cancer Foundation of America. http://www.Icfamerica.org.

2. The Cancer Genome Atlas. http://cancergenome.nih.gov/cancersselected.

3. Foundation Medicine. http://www.foundationone.com/.

4. Lung Cancer Profiles. http://www.lungcancerprofiles.com/.

5. Lung Cancer Mutation Consortium. http://www.golcmc.com/.

doi:10.1186/gm515

Cite this article as: Norris: Lung cancer patient advocacy and participatory medicine. Genome Medicine 2014 6:7. 